\title{
VARIABLES RELACIONADAS CON LA RECEPTIVIDAD DE LOS ESCOLARES ANTE UN PROGRAMA DE PREVENCIÓN
}

\author{
Manuel A. Sanabrla Carretero \\ Carlos Villanueva Gradín
}

\section{RESUMEN}

La prevención escolar plantea una serie de retos a la hora de aplicary explicar los resultados de estos programas, en el presente estudio se hacen una serie de reflexiones que tienen que ver con la metodología de aplicación y con las caracteristicas de los sujetos como aspectos determinantes de la receptividad y aprovechamiento de estos programas. Los datos que se ofrecen fueron obtenidos de una muestra de 5.066 escolares de 11-14 años que participaron en un programa de prevención de carácter universal. Los resultados indican que la implicación del sujeto en el consumo, el nivel de participación de la clase, las explicaciones del profesor y algunas consideraciones que valoran el no consumo como expresión de madurez e inteligencia resultan ser variables predictoras del aprendizaje y satisfacción con el programa.

Palabras clave: PREVENCIÓN ESCOLAR, EVALUACIÓN DE PROGRAMAS, VARIABLES DE MEDIDA, PREADOLESCENTES.

Realización del trabajo: El diseño, tratamiento estadistico, análisis de datos y redacción del presente estudio ha sido realizado en el Departamento de Investigación y Desarrollo de Programas de ESTINGA, S. L., empresa dedicada al desarrollo y aplicación de programas de prevención.

Nota: El Programa de Prevención en Drogodependencias CINENSINO se viene impartiendo en Galicia desde hace 5 años y está subvencionado por el Plan de Galicia sobre Drogas y Ayuntamientos.

Correspondencia:Carlos Villanueva Gradín. ESTINGA, S.L. Avda. del Camino Francés, $4-1^{\circ}$ A. 15704 SANTIAGO DE COMPOSTELA (Coruña). Tfno, 981577142 - Fax. 981 575603 - E-mail: clnensino estinga.com. 


\section{SUMMARY}

The school prevention raises a series of challenges at the moment of devoting and to explain the results of the preventive programs. In the present study there are a set of reflections related with methodology of application and with the characteristics of the subjects as relevant aspects of the acceptance and success of these programs. The information was obtained of a sample of 5.066 students (11-14 years-old) who took part in a program of prevention. The results show that main markers of prediction of success and satisfaction in the program are the commitment of student in the program, level of participation, explanations of teachers and insistence in interpreting abstention of use as expression of intelligence and maturity.

Key words: SCHOOL PREVENTION, EVALUATION OF PROGRAMS, MEASUREMENT OF VARLABLES, PREADOLESCENTS.

\section{INTRODUCCIÓN}

En numerosas investigaciones de los últimos años se ha venido informando de resultados muy dispares en relación con la eficacia de los programas de prevención escolar. Sin ignorar aquellos que informan de resultados satisfactorios son muchos también los que consideran que el impacto de los programas de prevención es limitado y que la efectividad tiende a decrecer en función de una serie de circunstancias de los escolares destinatarios que pueden ser determinantes de la eficacia de los mismos (Rooney y Murray, 1996; Bas, 2001; Baldivieso, 2004; Canning, Milward, raj y Warm, 2004).

No cabe duda que la prevención escolar exige una reflexión, ya que quizás no estemos haciendo verdadera justicia con todas las iniciativas preventivas que se llevan a cabo en los centros escolares dado que las evaluaciones que se hacen quizás estén mediatizadas por la influencia de una serie de circunstancias que se solapan con el efecto de los programas (García del Castillo, 2004). Una de esas 
circunstancias tiene que ver con las variables medidas para valorar el programa, ya que se ha observado en los estudios comparativos mediante meta-análisis que la eficacia de los programas suele ser mayor y más generalizada en aspectos que tienen que ver con la información, creencias y actitudes; siendo más pequeña en conductas de consumo (Ennett, Tobler y Ringwelling, 1994; Fernández, Nebot y Jané, 2002; Gómez-Durán, 2004).

En las valoraciones de los programas de prevención tendemos a hacer evaluaciones externas de carácter conductual, presuponemos que el alumno aprendió a generalizar y aplicar los conocimientos facilitados a situaciones de consumo en la vida real; sin embargo, hay que tener en cuenta que los mensajes preventivos emitidos desde los programas suelen ser acogidos desde posturas diferentes, a veces contrapuestas, por parte de los alumnos.

En nuestra experiencia preventiva en las aulas hemos detectado tres tipos de posicionamiento del alumnado. Una primera postura está representada por aquellos alumnos para los cuales el tema de las drogas resulta novedoso e interesante, son sujetos que han oído hablar de las drogas pero quizás sea esta la primera vez que el tema se aborda a nivel institucional con la suficiente seriedad y profundidad, lo que les permite descubrir cosas y contrastar otras ya conocidas por ellos, de manera que lo siguen con atención e interés. En la segunda postura está el alumnado que se muestra más escéptico, con posicionamientos cercanos a la neutralidad; no llegan ni mucho menos al rechazo, pero tampoco dan muestras de adhesión ante la información que reciben, como si interiormente estuvieran dudando y necesitasen corroborarto por ellos mismos sin descartar, por ejemplo, la auto-experimentación en un futuro próximo. La tercera postura engloba a un sector menos numeroso que da muestras claras de desacuerdo y rechazo del programa; son alumnos que se consideran la avanzadilla de la clase, para ello necesitan ir rompiendo moldes, son vistos por los demás con cierto respeto y admiración, están convencidos de que para hablar del consumo hay que tener experiencia o por lo menos posibilidad de estar cerca de aquellos que ya la tienen y la pueden facilitar. Sus intervenciones se encaminan a poner cuanto menos en duda lo que se les transmite desde el programa, calificándolo de teórico e incluso de alarmista, 
considerando sesgados y tergiversados los contenidos del programa. Así lo creen y con sus intervenciones en clase tratan de dejarlo claro para los demás, llegando en ocasiones a posicionarse como "saboteadores" de la propuesta preventiva que se les ofrece (Cánovas, 1994).

Es evidente que los adolescentes de hoy han asumido los beneficios y ventajas asociados al consumo, tanto es asi que algunos de ellos ya están consumiendo o a punto de hacerlo. Por tanto, el mensaje preventivo puede suponer un choque al cuestionar sus conocimientos, creencias, actitudes $\theta$ incluso conductas ya favorables al consumo (Zonabend, 1993; Funes, 1999; Mansilla y Vega, 1999; Sanabria y Villanueva, 2003). Si en el momento de recibir el programa tienen el convencimiento interno de que los beneficios que reporta el consumo compensan los posibles riesgos y están decididos a seguir coqueteando con las drogas, el programa preventivo puede verse como una amenaza para sus intenciones e intereses, pudiendo poner en marcha una serie de mecanismos tendentes a proteger sus intenciones y decisiones (Ordoñana y Gómez, 2002). De ser así en los escolares que mantienen algún tipo de consumo deberian aparecer muestras de reticencia o "resistencia" hacia la propuesta preventiva que les llega.

Aunque no está entre los objetivos de este estudio determinar las numerosas estrategias de "resistencia" que los sujetos consumidores pueden poner en práctica, pero algunos autores mencionan mecanismos como la "ilusión de la invulnerabilidad", se trata de un sistema organizado de creencias y actitudes mediante el cual los individuos implicados en el consumo tienden a considerar que tienen menos probabilidades que otras personas de que el consumo les cause daños o tenga para ellos consecuencias indeseadas; en el caso de haber algunos daños siempre serían de menor consideración que los que pueden afectar a otros, por tanto consumir para ellos no supone una amenaza inquietante (Rodin y Salovey, 1989; Zonabend, 1993; Becoña, 2002; Bersebé, Martínez y Tejeiro, 2002). Este mecanismo de defensa puede verse reforzado por otro que hace referencia a la "ilusión del control", tiene que ver con una sobreestimación de la propia capacidad para controlar el consumo en el momento que el sujeto lo desee y quiera, de manera que la 
capacidad de control se reduce a una mera decisión que se puede aplicar cuando se quiera (Van der Pligt, 1996; Ubillos, Páez, Mayordomo y Sánchez, 2003; Espada y Méndez, 2003).

Ya que los sujetos consumidores tienden a atribuir al consumo una serie de ventajas tanto en el disfrute de la sustancia como en la interacción social ligada al consumo, la oferta preventiva implica el cuestionamiento de esas consecuencias positivas. Cuando esto sucede, el programa preventivo puede provocar en estos sujetos un choque con cierta carga emotiva, pudiéndose incrementar en aquellos programas cuyo objetivo irrenunciable es conseguir la abstinencia total y absoluta de cualquier tipo de sustancia, tanto legal como ilegal, llegando a incorporar para ello mensajes alarmistas de connotaciones muy negativas que suelen producir un mayor efecto de rechazo entre ese sector de destinatarios (Beck, 1998; Ordoñana y Gómez, 2002).

Así pues, la mayor o menor proximidad de los sujetos respecto al consumo de sustancias legales se vislumbra como un aspecto importante a la hora de aplicar los programas de prevención a los escolares, desconocer esa situación puede minar su aplicabilidad, ya que de ello van a depender los contenidos del programa y el momento ideal para impartirlos, dependiendo de la edad de los sujetos (Chen y Zinder, 1996). Ese conocimiento es de gran importancia ya que puede llegar a condicionar los niveles de eficacia atribuidos al programa, si tenemos en cuenta que corı la edad también tiende a incrementarse la implicación de los escolares en conductas de consumo y el acceso a ellas cada vez se hace a edades más tempranas, sería necesario coordinar y ajustar la prevención al proceso evolutivo de los escolares para evitar desfases y zonas vaclas (Craig, 1996; Espada, Méndez e Hidalgo, 2000; Plan Nacional sobre Drogas, 2004). Algunos autores sugieren que la implantación de los programas de prevención de drogas de carácter universal debería hacerse antes de que los consumos de los escolares se manifiesten con cierta evidencia, sugiriendo que un buen momento podría ser el paso de la Educación Primarla (6 $6^{\circ}$ E.P.) a la Secundaria (12 E.S.O.), es decir el intervalo de edad que va de los 11 a los 12 años (Rooney y Murray, 1996; Bas, 2001; Becoña, 2002). Lo ideal sería, como indican Tobler y Stratton (1997), desarrollar programas ade- 
cuados para cada edad teniendo en cuenta las características evolutivas de los escolares y su implicación progresiva en el consumo.

Pero tan importante como los contenidos y actividades del programa son los responsables de su aplicación, los profesores que imparten los programas representan el canal de comunicación entre el mensaje y los receptores; su actuación pude determinar la viabilidad y eficacia de un programa o su inutilidad y fracaso. En este sentido, aspectos como la motivación, compresión de contenidos, el sentido de las actividades y generalización de aprendizajes, imprescindibles para que un programa funcione resulta importante su contribución; representan también un componente más de los programas de prevención escolar que no se debería ignorar.

Asl pues, considerando algunas de las cuestiones planteadas, centramos nuestra atención en una amplia muestra de escolares que participaron en un programa de prevención escolar con el fin de obtener datos que nos ayuden a ajustar las intervenciones futuras a las características de los alumnos, para ello nos planteamos los siguientes objetivos más concretos:

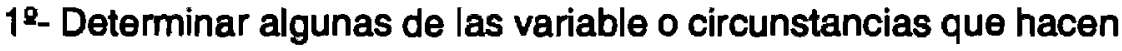
más sensibles y receptivos a los escolares ante la prevención de carácter universal.

$2^{2}$ - Ver si existe relación entre el aprendizaje y nivel de aceptación del programa con el grado de implicación de los sujetos en el consumo de sustancias legales.

$3^{2}$ - Valorar el papel que desempeñan los animadores del programa (profesorado) en cuanto a la receptividad e implicación de los escolares en los mismos.

\section{MUESTRA}

La muestra para el estudio fue seleccionada al azar haciéndose previamente una distribución proporcional por municipios en función del número de alumnos inscritos. De los 14.527 escolares participantes en el Programa se seleccionó una muestra de 5.066 sujetos, cuyas edades oscilaban entre los 11 -14 años, correspondiendo a los cursos de $1^{2}$ y $2^{2}$ de ESO. 
La mayoría de los alumnos implicados en el programa tienen entre 13-14 años y están cursando $2^{2}$ de ESO, siendo un $67,8 \%$ de la muestra; en cambio, los de 11-12 años, que cursan $1^{2}$ de ESO es un porcentaje inferior, concretamente el $32,2 \%$, lo que equivale a que dos de cada tres alumnos de la muestra son de $2^{2}$ ESO. Sin que fuera una pretensión exigida de antemano, la distribución de la muestra en función del sexo es equivalente. De los 5.066 escolares, la mitad, un $49,8 \%$, son varones y la otra mitad, un $50,2 \%$, mujeres.

\section{PROCEDIMIENTO}

Los sujetos en los que se basa el presente estudio han participado en un programa de prevención escolar de carácter universal denominado CINENSINO, llamado así porque se utiliza el cine como referencia para trabajar una serie de temas orientados a la prevención de drogas, más concretamente del tabaco y del alcohol. Para ello se le facilitan, después de ver la película, tanto a los profesores como a los alumnos, el material que constituye la Unidad Didáctica de esa película y que consta de un Manual del Profesor y un Cuaderno del Alumno, con una dedicación media de $\mathbf{4}$ horas por Unidad Didáctica y película. La labor preventiva se desarrolla en el aula, el profesor debe motivar, explicar, coordinar actlvidades, organizar los grupos de trabajo y concretar conclusiones elaboradas por los alurnnos. Para ello el quehacer de los alumnos se centra, en primer lugar, en un trabajo personal que se completa después en pequeños grupos y termina en una puesta en común con toda la clase. El programa consta de tres películas, cada una de ellas dedicada a un factor de riesgo / protección, concretamente fueron: "Un ocio juvenil en contextos de riesgo de consumo" con la película "Yamakasi" para la primera Unidad Didáctica, "creencias erróneas en el consumo de drogas" con la película "Corazones en Atlántida" para la segunda Unidad y "la conducta antisocial en el aula" con la película "Josie y las melódicas" para la tercera. Al profesor encargado de impartir el programa se le facilita un Curso de Formación vía internet.

Para evitar, en la medida de lo posible, que los alumnos se sintiesen directamente evaluados hemos tratado de integrar las 
preguntas en los contenidos de las unidades didácticas, dicha información era utilizada a la vez como material para trabajar y debatir en clase. Esas preguntas están recogidas y unificadas en el Anexo 1.

En algunos casos las preguntas se introduclan con la frase "Te agradeceríamos que nos ayudases a mejorar el programa respondiendo con sinceridad a estas cuestiones". Las preguntas incluyen una escala tipo Likert con 4 niveles eligiendo el valor más acorde con su situación y hacen referencia a la satisfacción con las actividades, a lo aprendido, a la implicación del profesor, al posicionamiento y rechazo del consumo. Los Cuadernillos, una vez trabajados por los alumnos, fueron recogidos por sus profesores y entregados a los responsables del programa. Prácticamente la totalidad de los centros educativos entregó el material, siendo muy pocos los alumnos a los que no se les pudo recoger por algún motivo.

\section{RESULTADOS}

\subsection{Grado de satisfacción y estimación del nivel de aprendizaje}

La estimación del grado de satisfacción se centró en las cuestiones y actividades propuestas en las Unidades Didácticas, pidiendo a los alumnos que valorasen en qué medida le gustaron, anotando en una escala de 4 valores (mucho, bastante, poco, nada). Los resultados indican un buen nivel de satisfacción, ya que para el $51 \%$ de la muestra, equivalente a 2.544 alumnos, las actividades han sido bastante satisfactorias y para un $24 \%$ (1.199 alumnos) las actividades fueron de mucho agrado. En cambio, para un $18 \%(920)$ resultaron de poco interés y para un $7 \%$ (365) no gustaron. Sumando los porcentajes podemos afirmar que las actividades relacionadas con la prevención del consumo de drogas propuestas en las Unidades, parecen ser de bastante y mucho interés para el $74 \%$ de los alumnos, despertando menos o poco interés para un $25 \%$.

Tratando de averiguar a qué tipo alumnos le gustaron más las actividades propuestas, relacionamos esta variable con el curso (19. $2^{2}$ ESO) y nos encontramos con que son los de $1^{9}$ de ESO los que 
muestran más satisfacción con las actividades, ya que a nivel proporcional el porcentaje de alumnos que le gustaron menos es menor y son más los que responden que le gustaron "bastante" o "mucho", tal como se puede apreciar por las alturas de las barras en la Gráfica 1. Los alumnos de $1^{\circ}$ de ESO superan en porcentajes a los de $2^{\circ}$ de ESO, $43 \%$ frente a $39 \%$ en "bastante" y $22 \%$ frente a $18 \%$ en "mucho"; en cambio, para los otros dos valores de la variable, "poco" y "nada", sucede lo contrario, los de $2^{\circ}$ ESO superan en porcentajes a los de $1^{\circ} \mathrm{ESO}$. De manera que el alumnado más joven de la muestra (11-12 años) es más receptivo con el programa y hace pensar en ellos como los destinatarios más idóneos a la hora de implantar el programa, coincidiendo también con lo ya han señalado otros autores (Rooney y Murray, 1996; Becoña, 2002).

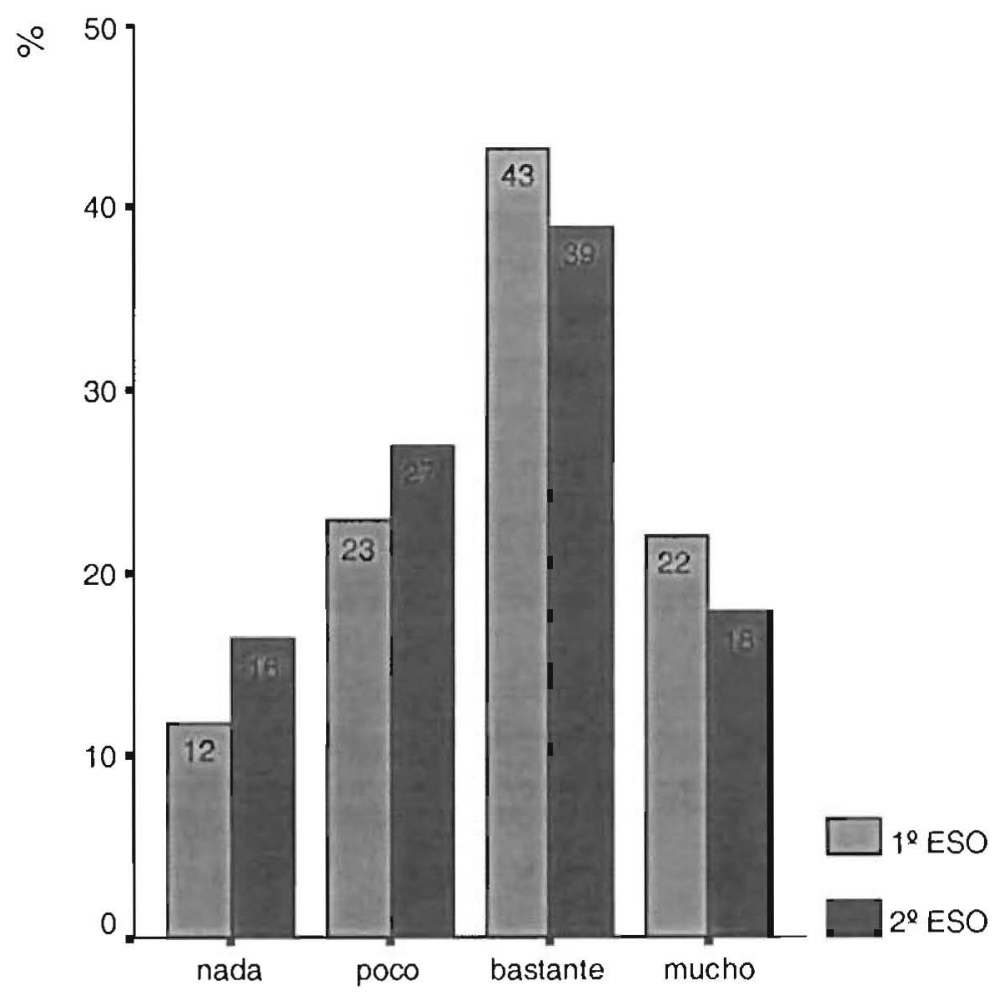

Gráfica 1.- Satisfacción con el programa según el curso 
Para estimar el nivel de aprendizaje que ha podido aportar el programa, se les preguntó a los alumnos acerca de su valoración sobre el mismo. Un $24 \%$ del alumnado (unos 1.178 sujetos) consideran que con las propuestas del programa han aprendido mucho en materia de prevención, el 53\% (2.645 sujetos) hacen también una buena estimación de su nivel de aprendizaje considerando haber aprendido bastante. Asi pues, con la suma de ambos tenemos que un $77 \%$ del alumnado de la muestra tienen la impresión de que el aprendizaje aportado por el programa es claramente satisfactorio. En cambio, un $17 \%$ de la muestra considera que ha aprendido poco y un $6 \%$ manifiesta no haber aprendido nada. Para tratar de clarificar algunas de las características que pudieran tener estos alumnos llevamos a cabo otros análisis adicionales. Mediante un análisis de correlación relacionamos los cigarrillos que los escolares habian fumado la última semana y la intención de fumar con el grado de satisfacción de las actividades y el nivel de aprendizaje provocado por el programa. Los resultados de esta exploración se recogen en la Tabla 1.

Tabla 1.- Relación entre consumo e intención versus satisfacción y aprendizaje

\begin{tabular}{|c|c|c|}
\cline { 2 - 3 } \multicolumn{1}{c|}{} & $\begin{array}{c}\text { Consumo cigarros } \\
\text { última semana }\end{array}$ & Intención de fumar \\
\hline $\begin{array}{c}\text { Satisfacción con las } \\
\text { actividades }\end{array}$ & $\begin{array}{c}r=-0.05 \\
p<0.01\end{array}$ \\
\hline $\begin{array}{c}\text { Estimación del } \\
\text { aprendizaje }\end{array}$ & $r=-0.47$ & $r=-0.10$ \\
$p<0.01$ & $p<0.01$ \\
\hline
\end{tabular}

Como se puede apreciar en la tabla, los resultados vienen a indicar con significación estadística que cuanta más intención de fumar tienen los escolares, el grado de satisfacción con las activi- 
dades del programa tiende a ser menor. Lo mismo se aprecia en cuanto a la percepción del aprendizaje emanado del programa, en la medida que dicho aprendizaje es más bajo o escaso la intención de consumir $(r=-0.10)$ y el consumo real $(r=-0.47)$ tiende a ser mayor; la relación inversa también se cumple, los mayores niveles de satisfacción y aprendizaje correlacionan de manera significativa con niveles significativamente más bajos de consumo. Así pues, los datos sugieren que cuanto más se fuma, menos se aprovecha y menos satisface el programa.

Pero aún podemos profundizar más en este aspecto, para ello dividimos la muestra en función del consumo, estableciendo dos grupos de alumnos, los que manifiestan haber fumado (Han fumado) y consumido alcohol (Han bebido) y los que nunca lo han hecho (Abstemios) y los comparamos mediante la prueba $t$ de contraste de medias obteniéndose los siguientes valores de la Tabla 2, todos ellos significativos.

Tabla 2.- Comparación entre abstemios y consumidores respecto a satisfacción y aprendizaje con el programa

\begin{tabular}{|c|c|c|}
\hline & Grado de satisfacción & Nivel de aprendizaje \\
\hline Han fumado & $t=-3.84$ & $t=-8.12$ \\
\hline Abstemios & $p<0.01$ & $p<0.01$ \\
\hline Han bebid & $t=-7.43$ & $t=-7.56$ \\
\hline Abstemio & $p<0.01$ & $p<0.01$ \\
\hline
\end{tabular}

Estos datos nos muestran que los consumidores de tabaco o alcohol y los abstemios de estas sustancias valoran de modo significativamente diferente tanto el grado de satisfacción con las actividades del programa como el nivel de aprendizaje que el pro- 
grama produce en ellos, de manera que los sujetos implicados en el consumo manifiestan menos satisfacción con las actividades del programa y dicen haber aprendido poco o nada con él. Parece pues, que la implicación del sujeto en el consumo resulta determinante para la receptividad y aprovechamiento del programa por parte de los destinatarios. Desde este punto de vista nuestros datos sugieren que los sujetos consumidores o con intención de hacerlo pueden ponen en marcha estrategias de resistencia que les hacen menos receptivos y en sus respuestas respecto al grado de satisfacción y aprendizaje así lo reflejan.

\subsection{Percepción sobre Informaclón de drogas}

Si los sujetos consumidores ponen en marcha ciertas estrategias de protección de su conducta e intención de consumir, la información de carácter preventivo emitida desde el programa debería ser un punto de desencuentro o elemento diferenciador entre sujetos abstemios y consumidores con criterios diferentes respecto a la receptividad y valoración de la misma. La pregunta que se les hizo hacía referencia a si el programa les habia ayudado a clarificar cosas sobre las drogas. Un $39 \%$ y un $26 \%$ consideran que el programa les ha ayudado bastante y mucho, lo que suma un total de $65 \%$ de alumnos que consideran que el programa les ha ayudado con su información a clarificar distintos aspectos relacionados con las drogas. En cambio, para el $19 \%$ la información le ayudó poco y para un $16 \%$ el programa no tuvo ninguna influencia como elemento clanificador.

Al hacer una comparación entre ambos cursos, son también los alumnos de $1^{\circ} \mathrm{ESO}$, los más jóvenes, los que consideran que han aprendido más acerca de las drogas, siendo también menor el porcentaje de alumnos de ese curso que dicen haber aprendido poco o nada, tal como se puede apreciar en los porcentajes de la Gráfica $2, y$ que de nuevo parece situar a los escolares de ese curso y tramo de edad (11-12 años) como los destinatarios más receptivos y aplicados. Estos datos son coherentes con lo apuntado en otros estudios indicando que a medida que aumentan las actitudes favo- 
rables al consumo y más se implican los escolares en el consumo real, cosa que evolutivamente progresa con la edad y cursos, aumentan las reticencias en un intento de justificar el consumo y evitar también disonancia cognitiva (Ubillos y col., 2003; Markham, Aveyard, Thomas, Charlton, López y Vries, 2004).

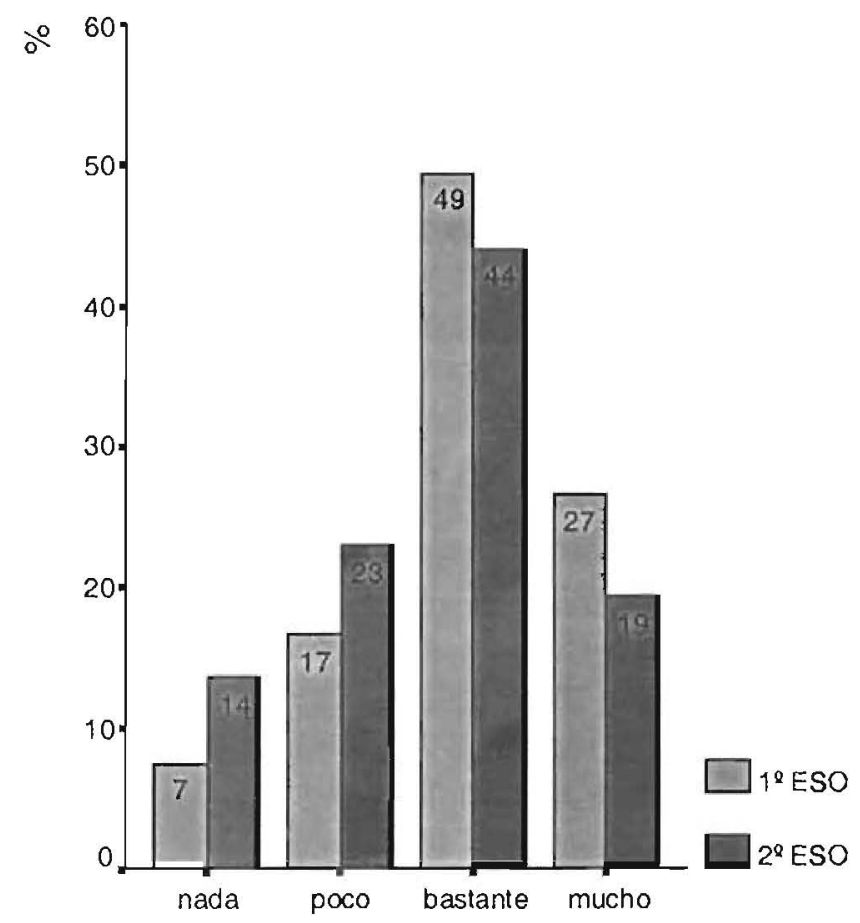

Gráfica 2. Información aportada por el programa en función del curso

\subsection{Papel del profesorado}

De cara a tener una estimación de ese aspecto hemos incluido una pregunta encaminada a obtener información de los alumnos respecto al nivel de explicaciones y aclaraciones dadas por el profesor en relación con los contenidos y actividades a realizar en las Unidades Didácticas. El $47 \%$ del profesorado ha facilitado explicaciones y aclaraciones a sus alumnos respecto a cuestiones de drogas 
y del programa, un $25 \%$ casi siempre también lo ha hecho; en cambio, un $14 \%$ y un $15 \%$ poco o nada lo ha hecho, lo que supone que un $29 \%$ del profesorado está muy poco implicado con el programa. Para concretar más su influencia del papel del profesor en la implementación del programa, realizamos una serie de análisis de tipo correlacional para determinar si afecta al grado de satisfacción del alumno, a su nivel de aprendizaje estimado y a la participación global de la clase. Los resultados de estos análisis los resumimos en la Tabla 3.

Tabla 3.- Relación entre explicaciones del profesor y repercusión en el alumnado

\begin{tabular}{|l|c|c|c|}
\cline { 2 - 4 } \multicolumn{1}{c|}{} & $\begin{array}{c}\text { Satisfacción con } \\
\text { las actividades }\end{array}$ & $\begin{array}{c}\text { Estimación del } \\
\text { aprendizaje }\end{array}$ & $\begin{array}{c}\text { Participación } \\
\text { de la clase }\end{array}$ \\
\hline EXPLICACIONES & $r=0.30$ & $r=0.31$ & $r=0.48$ \\
DEL PROFESOR & $p<0.01$ & $p<0.01$ & $p<0.01$ \\
\hline
\end{tabular}

Se puede observar en la tabla que todas las correlaciones son significativas, apreciándose una relación directa y positiva; de manera que cuanta más implicación del profesor, la incidencia del programa en los alumnos tiende a ser más satisfactoria, aprendiendo y participando más. Dato a considerar a la hora de implementar la prevención escolar, pues ellos mismos reconocen que no siempre están lo suficientemente preparados para impartirla y cuando lo hacen ni se le valora ni se le agradece su dedicación y trabajo, corriendo el riesgo de que la prevención se lleva a cabo con criterios de voluntariado sin que haya una sistematización y continuidad de la misma en los centros. 


\subsection{Predlcción del aprendizaje y la satlsfacclón con el programa}

Para tratar de identificar con mayor precisión el conjunto de variables que con más influencia inciden en la predisposición de los alumnos para aprovechar el programa de prevención, llevamos a cabo un análisis de regresión múltiple considerando como variables dependientes o a predecir la estimación del nivel de aprendizaje por parte del alumno y la satisfacción con las actividades del programa.

Así para la predicción del nivel de aprendizaje inducido por el programa, después de filtrar las variables no significativas, la ecuación de regresión quedó integrada por las siguientes variables todas ellas de carácter significativo: "Explicación de las actividades por parte del profesor", "consideración del no fumador como más maduro", "deseabilidad para salir con un chico/a que no fume", "consumo total alcohol al mes", "considerar la imagen del fumador de manera peyorativa", "consumo semanal de tabaco", "intención de fumar" y "considerar que fumar para ser adulto es una equivocación". El grupo de variables predictoras está integrado por una variable centrada en el contexto donde se lleva a cabo la prevención (explicaciones del profesor), otras cuatro referidas al ámbito de las creencias y atribuciones (consideración del no fumador como más maduro, deseabilidad para salir con un chico/a que no fume, considerar la imagen del fumador de manera peyorativa y considerar que fumar para ser adulto es una equivocación) y otras tres referidas al área adictiva (consumo mensual de alcohol, consumo semanal de tabaco e intención de fumar) (Ver Tabla 4)

De las 8 variables que conforman la ecuación de regresión cinco contribuyen a la ecuación de forma positiva, como se puede apreciar en los signos de beta y $t$ positivos, pero las que se integran en el área adictiva, es decir consumo de alcohol, consumo de tabaco e intención de fumar lo hacen con signo negativo, indicando una relación inversa respecto a la variable dependiente o a predecir, indicando que cuanto mayor es el consumo y la intención de fumar es predecible menores niveles de aprendizaje. Sin embargo, el conjunto de las 8 variables que entraron en la ecuación de regresión solo llegan a explicar más que el $12 \%$ de la varianza de la variable criterio, referida al nivel de aprendizaje del programa. Así pues el trabajo del profesor, 


\begin{tabular}{|c|c|c|c|c|c|c|c|c|}
\hline : & $\frac{N^{N}}{\sigma^{-}}$ & $\begin{array}{l}N \\
\stackrel{-}{*}\end{array}$ & $\stackrel{M}{N}$ & 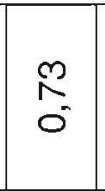 & $\begin{array}{l}\stackrel{M}{1} \\
\tilde{O}^{-}\end{array}$ & & & $\begin{array}{l}2 \\
\stackrel{2}{0}\end{array}$ \\
\hline 동 & 8. & 8 & $\bar{\delta}$ & 8 & 8 & 8 & $\begin{array}{l}\bar{\delta} \\
0^{\prime}\end{array}$ & : \\
\hline & $\begin{array}{l}\text { D } \\
=-\end{array}$ & 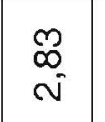 & $\begin{array}{l}\text { ले } \\
\text { N }\end{array}$ & $\hat{\sim}$ & $\begin{array}{l}\stackrel{M}{N} \\
\stackrel{N}{N}\end{array}$ & & 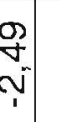 & N \\
\hline cy & $\begin{array}{l}\infty \\
0 \\
0\end{array}$ & $\frac{d}{0}$ & $\frac{0}{\frac{0}{0}}$ & & $\begin{array}{l}\infty \\
\frac{\infty}{\sigma} \\
0\end{array}$ & & 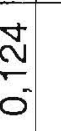 & $\frac{N}{0}$ \\
\hline एँ & $\begin{array}{l}\hat{N} \\
\text { O. }\end{array}$ & 'ò & $\begin{array}{l}8 \\
0\end{array}$ & & $\begin{array}{l}\mathscr{8} \\
0\end{array}$ & & $\begin{array}{l}0 \\
0 \\
0 \\
0\end{array}$ & - \\
\hline & - & $N$ & $m$ & $\nabla$ & (5) & $0 \mathrm{r}$ & & $\infty$ \\
\hline$\frac{\alpha}{\frac{\alpha}{\pi}} \frac{0}{\frac{\pi}{\pi}}$ & 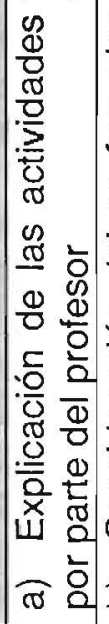 & 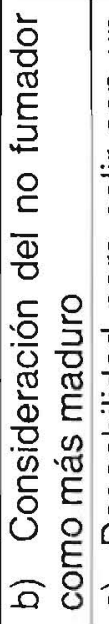 & 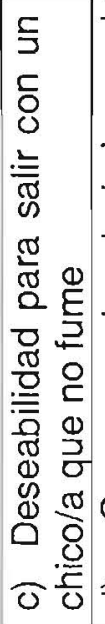 & 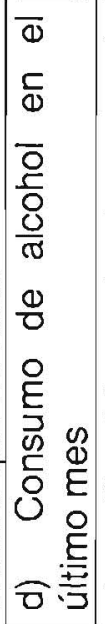 & 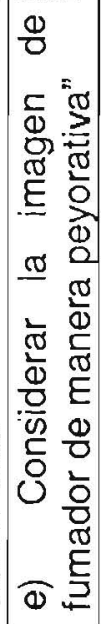 & 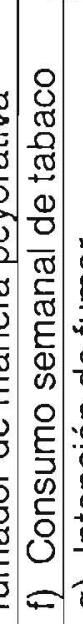 & 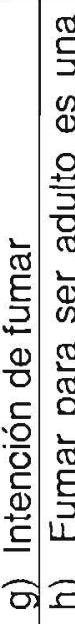 & 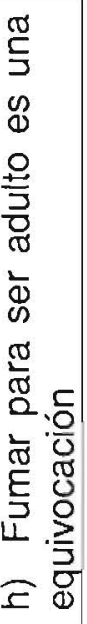 \\
\hline
\end{tabular}


la abstinencia del alumno, su intención de no fumar y ciertas creencias referidas a aspectos positivos asociados al no consumo son determinantes para que los programas causen aprendizajes preventivos en los escolares que los reciben.

Para completar el análisis consideramos también como variable a predecir el nivel de satisfacción que los alumnos manifestaron hacia las actividades del programa. Del conjunto de variables, las que resultaron con valor estadístico significativo fueron: "nivel de aprendizaje con las actividades", "nivel de participación de la clase" y "la consideración de más inteligente y sofisticado al no fumador" (Ver Tabla 5). Todas ellas son de signo positivo y en su conjunto explican el $29 \%$ de la varianza de la variable criterio centrada en la satisfacción con las actividades.

Tabla 5.- Variables predictoras respecto a la variable satisfacción con las actividades del programa

\begin{tabular}{|c|c|c|c|c|c|c|}
\hline Varlable Predictoras & P a so & B e fa & $\mathrm{B}^{2}$ & $t$ & $\begin{array}{l}\text { Nivel } \\
\text { de } \\
\text { signif. }\end{array}$ & $\begin{array}{l}\text { Error } \\
\text { estándar }\end{array}$ \\
\hline $\begin{array}{l}\text { a) Nivel de aprendizaje } \\
\text { con las actividades }\end{array}$ & 1 & 0,41 & 0,24 & 21,83 & 0.00 & 0,71 \\
\hline $\begin{array}{l}\text { b) Nivel de participación } \\
\text { de la clase }\end{array}$ & 2 & 0.21 & 0,28 & 11,31 & 0,00 & 0.69 \\
\hline $\begin{array}{l}\text { c) Consideracion de mas } \\
\text { inteligente y sofisticado } \\
\text { al no fum ador }\end{array}$ & 3 & 0.06 & 0,29 & 3.84 & 0.00 & 0,68 \\
\hline
\end{tabular}

\section{DISCUSIÓN Y CONCLUSIONES}

Para que todo programa de prevención resulte eficaz y responda a los fines para los cuales ha sido creado debe reunir una serie de condiciones y de características referidas a su estructuración y presentación para despertar el interés de los destinatarios, pero a 
parte de estos requerimientos existen otros aspectos referentes a los destinatarios de los programas que inciden significativamente en su aceptación y, en consecuencia, en la eficacia de los mismos. Conseguir un adecuado nivel de aceptación del programa puede ser el primero de los requisitos a conseguir aumentar la efectividad del mismo, para ello resulta interesante poder determinar los niveles de aceptación e indagar en las razones de los menos satisfechos para conseguir un mayor ajuste del programa a sus necesidades preventivas.

En la muestra de nuestro estudio hemos encontrado un $75 \%$ de alumnos para los cuales el programa parece ser de su agrado, en cambio el $25 \%$ restante no parece tan entusiasmado. Profundizando en las caracteristicas de los escolares menos satisfechos, se constata que está integrada por sujetos que manifiestan intención de consumir o están consumiendo ya, de manera que insatisfacción y proximidad al consumo van de la mano, siendo atribuible esta circunstancia a uno de cada 4 alumnos de la muestra. Existe, además, correlación de carácter significativo entre el nivel de aceptación del programa y la intención de consumir, por un lado, y el consumo real por otro; el sentido de la relación apunta a que cuanto más clara y segura es esa intención y más real el consumo las reticencias hacia el programa son más evidentes. En consecuencia, la situación de los escolares respecto al consumo de sustancias legales puede ser determinante para definir su receptividad respecto al programa. Quizás aquí se mezcien también factores relativos a la idiosincrasia de la adolescencia (rebeldía, rechazo, autoafirmación de la propia identidad frente a figuras adultas significativas, egocentrismo de las propias ideas y decisiones) que puede acrecentar las reticencias por ese tipo de iniciativas implantadas por adultos y autoridades académicas (Espada y Méndez, 2003).

Respecto a la percepción y asimilación de la información sobre drogas aportada por el programa, un $77 \%$ de los alumnos considera el programa de utilidad y ayuda; en cambio, un $23 \%$ tienen la impresión de que su nivel de aprendizaje ha sido escaso. En un análisis más minucioso del tipo y características de estos sujetos nos damos cuenta que los alumnos reticentes con el programa son los que menos han aprendido con él, mostrando una mayor intención o 
predisposición para consumir $(r=-0,10 ; p<0,01)$ y mayor consumo real $(r=-0,47 ; p<0,01)$. Esta relación llega a convertirse en una característica diferencial al comparar al grupo de alumnos que han consumido con los abstemios, observándose diferencias significativas entre ambos grupos respecto al grado de satisfacción y aprendizaje causado por el programa. El análisis de regresión nos permite concretar las variables que determinan estos dos aspectos, de dicho análisis resultó que la implicación del profesor, el nivel de participación de la clase, aspectos toxicológicos representados por el consumo de alcohol, de tabaco, la intención de fumar y ciertas consideraciones y creencias que valoran el no consumo y la imagen de abstemio resultan determinantes a la hora de predecir el aprendizaje generado por el programa y lo satisfechos que los alumnos se muestran con ét.

Estos datos nos hacen pensar que la proximidad al consumo y la experimentación con sustancias legales por parte de los escolares sean un lastre a la hora de implementar programas de prevención de carácter universal. Lo ideal sería poder filtrar a los escolares consumidores para encaminarlos hacia un programa selectivo especialmente adaptado a sus características y necesidades. El problema radica en que en el medio escolar es difícil hacer ese tipo de discriminación, ello implica sacar a la luz un tipo de conducta que los escolares suelen guardar con bastante intimidad, especialmente frente a los adultos, de hacerlo nos podemos enfrentar a problemas o cuanto menos mal entendidos entre profesores, padres y los propios alumnos. En la práctica real es muy frecuente que los programas de prevención escolar de carácter universal acojan a sujetos que están en situación de consumo. El aprovechamiento de este recurso por parte del alumnado consumidor va a ser escaso y pueden llegar a incidir de manera notoria en la efectividad del programa, especialmente si las variables de medida se centran en el área adictiva. Prescindir de ellos no resulta operativo ni a veces es posible (discriminación, intimidad, carencia de medios o programas altemativos de carácter selectivo, personal cualificado que imparta esos programas especificos, etc.) pero no hay que olvidar que entre un $4 \%$ $6 \%$ de los alumnos de 11-14 años son consumidores habituales de drogas legales y un porcentaje mayor tienen intención clara de hacerlo 
pronto (Plan Nacional sobre Drogas, 2004). Quizás en algunos de estos sutiles aspectos estén las claves que explicarían por qué la prevención universal no siempre muestra los resultados satisfactorios que cabría esperar en la reducción de los consumos.

Centrándonos en contexto escolar parece ser más conveniente y estratégico ofertar programas de prevención antes de que se manifiesten las primeras experiencias de consumo, quizás lo ideal sería iniciarlos en $6^{\mathfrak{Q}}$ de Primaria (11-12 años) ya que a medida que ascienden en cursos las probabilidades de iniciarse en el consumo aumentan y las probabilidades de atajarlo directamente disminuyen. Si tenemos en cuenta que el consumo tiende a aumentar entre los escolares y cada vez ocurre a edades más tempranas y que la incorporación de mujeres adolescentes en el consumo de sustancias, especialmente las legales tiende a ser mayor, parece evidente que a la prevención le tocará mover ficha en este campo si queremos incidir de manera más eficaz en aquellos sujetos que más la necesitan (Rooney y Murray, 1996; Espada, Méndez e Hidalgo, 2000; Femández, Nebot y Jané, 2002).

No cabe duda que en este campo todavía tenemos que seguir avanzando, tiene plana vigencia lo señalado por Aubá y Villalbi (1990) al afimar que siguen exlstiendo dudas en cuanto a la población diana objeto de intervención preventiva, de su situación respecto al consumo, de los aspectos referentes a los contenidos y de los aplicadores de los programas. Habría que añadir que las decisiones sobre la mayoría de estos aspectos las asume el personal directivo de los centros sin contar muchas veces con los técnicos de prevención; de suerte que la prevención inadecuada en momentos inoportunos quizás sea algo más que un hecho aislado en el medio escolar.

Si algunos escolares dan muestras de recelo ante una iniciativa de prevención quizás lo sea por poner en tela de juicio muchas de sus decisiones y posturas ya consolidadas a favor del consumo, señal evidente de que la iniciatlva ha llegado demasiado tarde. EI adolescente evalúa la intervención que recibe en función de la susceptibilidad que el objeto de amenaza (droga) entraña para él en ese momento, es decir, qué probabilidad atribuye a que le afecten los riesgos predicados por la acción preventiva (Mansilla y Vega, 
1999). Es evidente que para los jóvenes las consecuencias del consumo son vistas a muy largo plazo, con frecuencia se imponen las ventajas del consumo sobre los inconvenientes del mismo, de suerte que los riesgos son percibidos como algo trivial $\theta$ irrelevante, para el alumnado adolescente la intervención preventiva podría carecer de utilidad inmediata (Witte y Allen, 2000). También habría que considerar la influencia de otras circunstancias que actuarían de colchón amortiguador de la incidencia del programa, se trata de variables de carácter cognitivo relativas a creencias y actitudes que tienen mucho que ver en la percepción de los riesgos asociados al consumo adolescente, tales como la "ilusión de la invulnerabilidad" (Bersebé, Martínez y Tejeiro, 2002) y la "ilusión del control" (Ubillos y col., 2003; Espada y Méndez, 2003).

Partiendo de estas posturas cabe esperar que la respuesta iricial de los escolares ante una propuesta de prevención puede oscilar entre dos extremos y en función de la proximidad o lejanía de los sujetos respecto al consumo, condicionando su respuesta receptiva hacia el programa y lo aprendido del mismo (Ordoñana, Gómez y Galván, 2000; Donovan y Henley, 2000). Estas diferencias entre actitudes receptivas por un lado y de reticencia por otro, son clave para la implementación de los programas preventivos, ya que si el programa provoca en el sujeto una respuesta defensiva se corre el riesgo de que centre su atención en los aspectos emocionales que la iniciativa genera en él, vivenciándola como algo amenazante que quizás vaya a rechazar o simplemente ignorar (Ordoñana y Gómez, 2002). En este sentido se han sugerido distintas iniciativas cuya finalidad es hacer más receptivos a los alumnos consumidores. Flórez (2003) habla de estrategias encaminadas a reducir los beneficios asociados al consumo de sustancias legales, estrategias encaminadas incrementar la percepción de los costes y el acceso a los beneficios del no consumo. Otros autores abogan por incluir mensajes claros en los programas preventivos encaminados a reconocer el consumo de los jóvenes, sustituyendo mensajes radicales por otros referentes al consumo responsable, incluyendo propuestas entre el uso, el abuso y la abstinencia, ya que se ha observado que pretender alcanzar la abstinencia total puede reducir la capacidad de maniobra de muchos 
jóvenes que consumen, al considerar que el programa se orienta a evitar un paso que ellos ya han dado, el de consumir, situándose al margen de la iniciativa de prevención (Fernández, Nebot y Jané, 2002).

Por otra parte, si en los programas de prevención escolar de carácter universal no resulta aconsejable ni es operativo excluir a los consumidores y se les hace un hueco en ellos, debemos ser prudentes y operar con cierta cautela a la hora de valorar la eficacia de esos programas, ya que la condición de consumidor, según nuestros datos, determina la receptividad y aprovechamiento del programa. Estos aspectos también se deben tener en cuenta a la hora de decidir el momento más adecuado para poner en marcha una iniciativa de prevención, teniendo en cuenta la situación de los escolares ante el consumo, de ello dependerá el hacer la prevención en el momento más adecuado y efectivo.

Finalmente, la implicación del profesorado es una variable de peso en el éxito del programa, siendo quizás la circunstancia que mejor explique el aprovechamiento del mismo por parte de los alumnos. El hecho de que, según nuestros datos, haya un sector del profesorado (29\%) poco motivado y con escasa implicación en la buena aplicación del programa es determinante para la eficacia del mismo. El contar con el profesorado del centro como primeros responsables de la aplicación de los programas preventivos siempre se ha considerado como una ventaja; el conocimiento que tienen de sus alumnos, su capacidad de organización son puntos a favor. No obstante, existen otros aspectos como la propia problemática de los centros educativos, pérdida de rol del profesorado, sobrecarga de trabajo, falta de interés, el tener que impartir un programa ajeno a su especialidad o perfil pedagógico, la ausencia de reconocimiento y remuneración por un trabajo adicional pueden ser algunos de los inconvenientes que mermen la efectividad de la prevención ejecutada por el personal docente del propio centro (Bas, 2001). Serfa también un aspecto a considerar en el futuro para tratar de potenciar la eficacia de los programas con medidas tendentes a preparar a los profesores en tareas de prevención, la figura del profesor especialista centro, tras un período de formación podría ser una buena alternativa. 


\section{BIBLIOGRAFIA}

Aubá, J. y Villabi, J. (1990). Prevención desde la escuela del uso del tabaco y de otras sustancias adictivas. Gaceta Sanitaria, 17, 70-75.

Baldlvleso, L. (2004). ¿Ha fracasado la prevención sobre drogas? Evaluación de un modelo. Ponencia. $1^{2}$ Congreso Iberoamericano de Trastornos Adictivos. Santiago de Compostela, 26-28 Febrero, 2004. WWW. Conductasadictivas.org.

Bas, E. (2001) Prevención de drogodependencias en secundaria. Ed. Narcea. Madrid.

Beck, J. (1998). 100 year of "just say no" versus "just say know". Reevaluating drug education goals for the coming century. Evaluation Review, 1, 15-45.

Becoña, E. (2002). Bases cientificas de la prevención de las drogodependencias. Madrid. Plan Nacional sobre Drogas.

Bersebé, R., Martínez, R. y Tejelro, R. (2002). Los que se arriesgan, ¿saben cuánto se arriesgan? Apuntes de Psicología, 20, 3-16.

Canning, U., MIIIward, L., RaJ, T. y Warm, D. (2004). Drug use prevention among young people: A review of reviews. London. Health Development Agency.

Cánovas, G. (1994). Adolescentes y alcohol. Bilbao. Ed. Mensajero.

Cralg, G.J. (1996). Desarrollo psicológico. (7 Ed.). México. Prentice-Hall Hispanoamericana.

Chen, K. y Zlnder, A. (1996). When is the critical moment to provide smoking education at schools?. Journal of Drug Education, 16, 121-133.

Donovan, R. J. y Henley, N. (2000). A conceptual framework for fear arousal and threat appeals in health promotion communications. Health Promotion Journal of Australia, 10, 84-88.

Ennett, S., Tobler, N. y RIngwelling, R. (1994). How effective is drug abuse resistance aducation?. A meta-analysis of proyect DARE outcome evaluations. American Joumal of Public Health, 84, 1394-1401.

Espada, J. y Méndez, F. (2003). Prevención del abuso de alcohol y del consumo de drogas de síntesis. Madrid. Ed. Pirámide.

Espada, J.; Méndez, F. e HIdalgo, M. (2000). Consumo de alcohol entre escolares: Descenso de inicio y cambios en los patrones de ingesta. Adicciones, $12,57-64$.

Fernández, S.; Nebot, M. y Jané, M. (2002). Evaluación de la efectividad de los programas escolares de prevención del consumo de tabaco, alcohol y cannabis: ¿Qué nos dicen los meta-análisis? Revista Española de Salud Pública, 76, 175-187.

Flórez, L. (2003). Diferencias asociadas al género en los argumentos, las motivaciones y la autoeficacia ante el consumo de alcohol en estudiantes universitarios de Bogotá. Revista Peruana de Drogodependencias, 1, 173-192. 
Funes, J. (1999). Diversión y uso de drogas. Proyecto, 32, 17-20.

García Del Castillo, J.A. (2004). Problemas y límites asociados a la evaluación de la prevención de drogas. Ponencia. $1^{2}$ Congreso lberoamericano de Trastornos Adlctivos. Santiago de Compostela, 26-28 Febrero, 2004. WWW. Conductasadictivas.org.

Gómez-Durán, B. (2004). Evaluación de los programas preventivos. CINENSINO (Coord.). Una propuesta teórica y metodológica para la prevención del consumo de drogas (pp. 97-110). Colección Drogodependencias, 32. Santiago. Xunta de Galicia.

Mansllia, F. y Vega, P. (1999). Hipótesis de la susceptibilidad en drogodependencias. Reflexiones para un modelo etiologico. Revista Española de Drogodependencias, 24, 17-30.

Markhan, W.; Aveyard, P.; Thomas, H.; Chartton, A.; López, M. y Vrles, H. (2004). What determines future smoking intention of 12-13 years old UK, African-Caribbean, Indian, Pakistani and white young people? Health Education Research, 19, 15-28.

Ordoñana, J. R. y Gómez, J. (2002). Amenaza y persuasión en mensajes de salud sobre consumo de alcohol: de la teoría a la práctica. Adicciones, $14,479-485$.

Ordoĥana, J. R.; Gómez, J. y Galván, F. (2000). El uso del miedo en los mensajes de salud. Gaceta Sanitaria, 14, 45-59.

Plan Nacional Sobre Drogas (2004). Estadísticas de consumo. Plan Nacional sobre Drogas. Madrid. Ministerio del Interior.

Rodin, J. y Salovey, P. (1989). Health pschology. Annual Review of Psychology, 64, 243-256.

Rooney, B y Murray, D. (1996). A meta-analysis of smoking prevention programmes after adjustement for errors in the unit of analysis. Health Education Quarterly, 23, 48-64.

Sanabrla, M. A. y Villanueva, C. (2003). Creencias y convencionalismos relacionados con el consumo de tabaco en preadolescentes. Revista Española de Drogodependencias, 28, 218-244.

Tobler, N. y Stratton, H. (1997). Effectiveness of school-based drug prevention programmes: a meta-analysis of the research. The Joumal of Primary Prevention, 18, 71-128.

Ubillos, S., Páez, D., Mayordomo, S. y Sánchez, F. (2003). Sesgos cognitivos y explicaciones asociadas como factores determinantes de las conductas sexuales de rlesgo. Revista de Psicología Social, 18, 261-279.

Van Der Pllgt, J. (1996). Risk perception and self-protective behavior. European Psychologist, 1, 34-43.

Witte, K. y Allen, M. (2000). A meta-analysis fear appeals: Implications for public health campaigns. Health Education and Behavior, 27, 591-615.

Zonabend, F. (1993). Au pays de la peur deniée. Communications, 57, 121130. 


\section{ANEXO 1}

1. Curso

2. ¿Has fumado alguna vez?

3. ¿Cuántos cigarrillo has fumado en el último mes?

4. ¿Cuántos cigarrillo has fumado en la última semana?

5. Cuántas veces te hubiera gustado fumar, aunque de hecho no lo hicieras, en las últimas dos semanas:

Ninguna Alguna Varias Bastantes Muchas

6. ¿Has probado algún tipo de bebida alcohólica? Sí No

7. En el último mes, ¿qué tipo de bebida consumiste?

$\begin{array}{lll}\text { Cerveza: } & \text { Ninguna Alguna vez } & \text { El fin de semana } \\ & \text { Todos los días Más de una vez al día } \\ \text { Vino: } & \text { Ninguna Alguna vez } & \text { El fin de semana } \\ & \text { Todos los días Más de una vez al dia } \\ \text { Combinados: } & \text { Ninguna Alguna vez } & \text { El fin de semana } \\ & \text { Todos los días Más de una vez al dla } \\ \text { "Chupitos": } & \text { Ninguna Alguna vez } & \text { El fin de semana } \\ & \text { Todos los días Más de una vez al día } \\ \text { Otros: } & \text { Ninguna Alguna vez } & \text { El fin de semana } \\ & \text { Todos los dlas } & \text { Más de una vez al dla }\end{array}$

8. Valora el grado de importancia que tienen estas afimaciones para ti

8.1 Tu valla personal aumenta cada vez que consigues no fumar un cigarrillo que te ofrecenr"

Nada importante Algo Bastante Muy importante

8.2 "Es más deseable salir con un chico/a que no fume" Nada importante Algo Bastante Muy importante

8.3 "La gente considera que los que fuman tienen mala imagen" Nada importante Algo Bastante Muy importante

8.4 "La gente tiende a ver cada vez más a los chicos/as que no fuman como más maduros"

Nada importante Algo Bastante Muy importante 